\title{
ESTRATEGIAS DE SOSTENIBILIDAD EN CIUDADES FRONTERIZAS AFECTADAS POR LA MIGRACIÓN. EL CASO DE LA CIUDAD DE MARACAIBO.
}

\author{
Fernando Torres Granadillo* \\ http://orcid.org/0000-0003-3464-7876 \\ Morela Pereira Burgos \\ http://orcid.org/0000-0003-4331-1530
}

RECIBIDO: Agosto 2019 / ACEPTADO: Marzo 2020 / PUBLICADO: Mayo 2020

\begin{abstract}
Como citar: Torres Granadillo, Fernando; Pereira Burgos, Morela (2020). Estrategias de sostenibilidad en ciudades fronterizas afectadas por la migración. El caso de la ciudad de Maracaibo. Telos: revista de Estudios Interdisciplinarios en Ciencias Sociales, 22 (2), Venezuela. (Pp.342-356).

DOI: www.doi.org/10.36390/telos222.07
\end{abstract}

\section{RESUMEN}

El presente trabajo analiza el problema de la inflación desde la perspectiva de la Escuela Austriaca (EA), pues aun cuando se le conceptúe como "antigua", pues data de finales del siglo $\mathrm{XIX}$ y principios del XX, tiene una explicación respecto a este y otros problemas macroeconómicos. Destacan autores como. Este paradigma se enriqueció con los aportes de Mises (1934) con Teoría del Dinero y el Crédito, Hayek (1985) con La Desnacionalización del Dinero, Ropke (1960) con A humane Economy y Rothbard (1990) con Teoría austriaca del dinero. Sin embargo, también se hace alusión respecto a esta problemática por parte de los autores de la denominada corriente principal. Se recurre a información de tipo cualitativo y cuantitativo. Como resultado de este trabajo se señala que, aunque las demás corrientes de pensamiento económico no difieren mucho en señalar los efectos de la inflación para la sociedad, la Escuela Austriaca, identifica como causas a la expansión crediticia y al déficit y deuda pública creciente. Como hallazgos derivados de la revisión de la literatura deben señalarse al creciente intervencionismo estatal, fuertes expansiones en el crédito, distorsiones en los precios y la presencia de ciclos de expansión y contracción en la economía como regularidad. De esto modo, se concluye que las instituciones financieras y políticas deben velar por el crecimiento económico con estabilidad por el bien de la sociedad.

Palabras clave: Inflación, ciclo económico, políticas de estabilización, Escuela Austriaca.

\footnotetext{
Doctor en Ciencias Humanas. Profesor Titular de la Universidad del Zulia. Correo electrónico: ftorresgranadillo@gmail.com

Doctora en Ciencias Sociales, mención Gerencia; Magister en Gerencia de Empresa, mención Servicios Administrativos: Licenciada en Administración Comercial; Técnico Superior en Administración. Profesora titular a dedicación exclusiva en la Facultad de Ciencias Económicas y Sociales (FCES) de la Universidad del Zulia (LUZ). Investigadora del Centro de Estudios de la Empresa de FCES-LUZ. Acreditada investigadora nacional desde 2001. Miembro de la Red de investigación sobre Gobernanza asociada al equipo de investigación Gobernanza Universitaria en Iberoamérica de la Universidad de los Lagos en Chile. Maracaibo, Venezuela.

Correo electrónico: morela.pereira@gmail.com

https://scholar.google.com/citations?user=XrCG2H0AAAAJ\&hl=es

https://www.researchgate.net/profile/Morela Pereira2
} 


\section{Sustainability strategies in border cities affected by migration. The case of the city of} Maracaibo.

\section{ABSTRACT}

The objective of the research was to design a sustainable territorial planning model for local border spaces, with the condition of diaspora situations. The theoretical basis corresponds, among other authors, to the approaches of Arango (1985), Massey (1998), Foucher (1991), Lin (2001), Burt (2004), Torres (2011) y Vázquez Barquero (1988). The research of analytical, explanatory and projective nature made use of various documentary and field techniques, useful for the design of a theoretical-operational model that responded to the realities of local bordertype environments, on the network of factors that can influence the levels of social-economic development, when these spaces are affected by diaspora conditions. Using inferential abduction, deduction and induction as logical reasoning methods, it was theoretically validated and empirically contrasted. The results show that local economic development (Maracaibo) definitely contrasts with economic development at the country level, accentuating the elements of the territory and their effects on economic, technological, political and social dimensions. It concludes about the need to relocate the concept of development in a constructivist, subjective and intersubjective framework, and, by the way, endogenous. Based on these results, a territorial and sustainable development planning model is proposed for border cities that can manage migratory movements, designed based on a conception of concentric and reticular rings, with a high porosity to facilitate dynamic relationships, and especially open, among the participating social agents, for the exchange of information with added value content.

Keywords: sustainability strategies, territorial development, migratory flows, border, diaspora.

\section{Introducción}

Los procesos y acciones para la sostenibilidad del desarrollo en ciudades con inteligencia territorial exigen, entre algunas condiciones, disposición activa de los gobiernos locales con mensajes de confianza a los agentes sociales, incentivos a las relaciones interorganizacionales e interconectividades en disposición de retículas como estrategias de acción local, homogeneidad de información, administración transparente de los recursos y equidad en la distribución de responsabilidades, esfuerzos y beneficios.

Dichas condiciones, exigen replanteamientos en el caso de ciudades fronterizas con flujos permanentes de recursos socio-productivos y poblaciones flotantes con identidades propias y diferenciadas del resto de ciudades nacionales. En el caso de situaciones de diáspora, se han establecido planes que permiten hacer de las contingencias, modos de actuación regulares. Los mayores desafíos de la planificación territorial surgen ante inéditos movimientos poblacionales que abren rutas desconocidas y desbalancean el sistema fronterizo y sus mecanismos históricos de autorregulación.

La diáspora ha llegado a un país sin antecedentes contemporáneos: Venezuela, agravando su situación interna y alterando sorpresivamente las dinámicas de diversas naciones. ¿Cómo se configuran los sistemas socio-productivos de las ciudades fronterizas ante la vorágine de la diáspora venezolana?, ¿Cuáles son las incidencias en los planos social, político, 
económico, entre otros, necesarios para la viabilidad de las ciudades fronterizas?, ¿Cómo son las afectaciones en las fuerzas dinamizadoras del desarrollo territorial ante el fenómeno de la diáspora? Son algunas de las rutas en la búsqueda de respuestas y la generación de acciones que indiquen señales coherentes para disminuir los actuales niveles de entropía.

El objetivo de la investigación fue diseñar un modelo de planificación territorial sostenible para espacios locales fronterizos, con el condicionante de situaciones de diáspora. Desde la perspectiva metodológica, se trata de una investigación de naturaleza analítica, explicativa y proyectiva. Se hizo uso de diversas técnicas documentales y de campo, útiles para el diseño de un modelo teórico-operativo que respondiera a las realidades de los entornos locales de tipo fronterizo, sobre el entramado de factores que pueden incidir en los niveles de desarrollo económico-social, cuando dichos espacios están afectados por condiciones de diáspora. Utilizando la abducción inferencial, la deducción e inducción como métodos de razonamiento lógico, se validó teóricamente y contrastó empíricamente.

\section{La madeja de la migración}

El mundo en sus composiciones poblacionales manifiesta los matices de la migración. Es posible asumirla como un proceso que acompaña el devenir histórico de la humanidad con alcances a lo largo del horizonte temporal. Allí radica la base de un carrete o madeja que cuando comienza a desenrollarse se expande $y$, al mismo tiempo, abre diversas vertientes de interpretación y análisis. Pudiera inferirse que en esta postmodernidad, las perspectivas se han multiplicado, además de complejizarse debido a los cambios ontológicos sobre el mundo y la humanidad; los movimientos geopolíticos, la fragmentación de mercados y diferenciación de las personas como consumidores, el internet, la emergencia de las redes sociales, la marcada onda expansiva de las tecnologías de información y comunicación, entre otros factores.

Dichos aspectos modelan nuevas formas interpretativas acerca de la migración. Sobre todo como un proceso que puede mutar entre lo individual y lo colectivo; incidentes en las relaciones sociales y las relaciones interorganizacionales. El análisis y comprensión de la migración es una tarea compleja, que se transforma en el tiempo por las diferentes modalidades que adquiere, esto en virtud de los fenómenos multidimensionales que impactan y motivan al migrante (Fernández, 2013).

De esta manera, las guerras y conflictos sociales, los regímenes totalitarios y persecuciones; las crisis económicas y hambrunas, como es conocido, tienen afectaciones directas y más que proporcionales en las diásporas. Pero también, la persecución étnico 0 religiosa, el mejoramiento de la calidad de vida, la búsqueda de desarrollo individual o familiar, oportunidades de empleo y educación, el acceso a bienes y servicios, entre otras (Aruj, 2008)

Con base en estas consideraciones, la investigación se apalanca en la definición de migraciones propuesta por Arango (1985), quien las presenta como traslados de domicilio a cierta distancia, relativamente permanente o con cierta voluntad de permanencia. Fortalecida con la perspectiva de Blanco (2000), que asume tres subprocesos que abarca el espectro migratorio: espacial, el movimiento ha de producirse entre dos delimitaciones geográficas significativas (como son los municipios, las provincias, las regiones o los países); temporal, el desplazamiento ha de ser duradero, no esporádico; social, el traslado debe suponer un cambio significativo de entorno, tanto físico como social. 
No obstante, tanto la teoría neoclásica, la teoría de los factores push-pull y la teoría con perspectiva histórica-cultural, así como las teorías sobre la perpetuación de los movimientos migratorios y la globalización contienen componentes de utilidad en las explicaciones que se generan actualmente. De la teoría de los factores push-pull, son apropiados sus explicaciones acerca de las condiciones que empujan (push) a migrar cuando los miembros de una localidad, región o país, compararan sus niveles de bienestar con otras condiciones más ventajosas que existen en otros lugares, las que ejercen una fuerza de atracción (pull), generándose de esta manera, una dinámica de expulsión (push) y atracción (pull) (Massey, 1998). El fenómeno migratorio no solo altera los espacios como estancos sino que modifica las dinámicas socioproductivas en especial de los ámbitos fronterizos y en especial de las ciudades irradiadas por las fronteras.

\section{La migración venezolana. Una mirada hacia lo inédito y explosivo}

El crecimiento de la industria petrolera rompió las tendencias de comienzos del siglo XX que condenaban al país a vivir en la pobreza. Se aceleró el proceso de acumulación de capital, urbanización y expansión del mercado interno. La captación de una renta internacional a partir de la venta de petróleo, sin contrapartida de esfuerzo nacional, generó elementos característicos: mayor importancia al empleo público; incremento de la demanda de bienes y servicios inducida por el gasto público; baja presión tributaria para financiar las actividades propias del Estado; estructura productiva desbordada en los servicios, es decir, la existencia de un ingreso adicional por la renta, sin contrapartida en la producción de bienes obligó a que se desarrollen las actividades de servicios públicos, comercio, transporte, almacenamiento y actividades financieras para distribuir las importaciones que estimula la renta.

De acuerdo con las cifras presentadas en los informes económicos del Banco Central del Venezuela, en el período 1950-1977 el PIB per cápita creció a la tasa media anual del 3,2 $\%$. En 1977 alcanzó su valor máximo para luego iniciar un proceso de declinación, apenas con leves de aumentos, que de ninguna manera dejan dudas sobre la clara tendencia decreciente del PIB per cápita. La brutal caída de la inversión, sobre todo de la privada, que se manifestó desde la década de 1980 y que se tradujo en la tendencia decreciente de la densidad de capital por trabajador, evidenció el agotamiento del modelo basado en el reparto de la renta petrolera, (Baptista, 2005).

La tendencia decreciente se amortiguó por el alto crecimiento del PIB per cápita del lapso 2003-2008, años de bonanza petrolera, el cual fue de 8,5\% como promedio anual. Tal crecimiento se explica fundamentalmente por la fuerte expansión del gasto público, que a su vez tuvo origen en la bonanza petrolera que se vivió en ese período. La crisis económica mundial que se inició en 2007 y reapareció en 2009. Venezuela, con un contexto institucional de baja calidad, con políticas que exacerban los desequilibrios macroeconómicos y desestimulan la inversión privada, no tiene condiciones de base para crecer sostenidamente porque carece del vigor que transmite la sostenida acumulación de capital, especialmente del sector privado.

Por otra parte, la polarización política, la concentración de poderes en la estructura de gobierno y la pérdida progresiva de libertades comenzaron a minar la estabilidad social venezolana. Una especie de desesperanza colectiva comenzó a incubarse hasta alcanzar la magnitud de una figura de mil cabezas. La formación de un complicado panorama que proyecta, 
Estrategias de sostenibilidad en ciudades fronterizas afectadas por la migración. El caso de la ciudad de Maracaibo

para la mayoría de los venezolanos, un nudo gordiano; una suma de situaciones agobiantes que empujan -push- a movimientos migratorios por escape.

Aun cuando los movimientos migratorios derivados de la situación descrita se iniciaron desde hace más de diez años, su agudización se ha manifestado desde los últimos cuatro años, sin lograr estabilizar los niveles de conflicto social de Venezuela como país de origen, como planteó Aruj (2008); sino por el contrario, expandiendo la conflictividad en lo político, económico y social a los países receptores, aumentando la competencia laboral, profundizando los círculos de pobreza, incrementando la xenofobia y la persecución.

En el caso de Venezuela, la predisposición cultural instaurada por su bonanza petrolera, lo hizo un país receptor de inmigrantes. Sin mayor experiencia migratoria ni enlaces de contacto y precarias redes sociales, el costo de aprendizaje migratorio comenzó a ser asumido al ser comparado en términos personales, familiares y colectivos con el desbordamiento de inseguridad, pobreza, desempleo, pérdida de integridad de vida por alimentos y medicamentos, inhabitabilidad, problemas de educación, ineficiencia de servicios públicos, entre otros.

La efervescencia de la diáspora venezolana en la actualidad supera los registros formales de las organizaciones asociadas con los flujos migratorios. Tienden a ocurrir "comportamientos cumbres" o explosivos derivados de la polarización política en momentos álgidos y el deterioro a niveles erosivos vitales para la población. Las cifras expost reflejan una porción de lo que acontece. Los estratos socio-económicos en mayores condiciones de pobreza constituyen flujos migratorios de difícil registro. De acuerdo a cifras de la Oficina Internacional para las Migraciones (OIM), organismo de la Organización de las Naciones Unidas y la Agencia de la ONU para los Refugiados (ACNUR), el número de refugiados y migrantes de Venezuela en todo el mundo alcanzó a mediados del 2018, los tres millones de personas. La tendencia migratoria es ascendente y las diversas proyecciones se acercan a representar en un lapso no mayor a dos años, el $10 \%$ de la población venezolana.

Lo inédito de la emigración ha originado que sus alcances apenas comiencen a ser registrados con fines de interpretación para intentos de ordenamiento. Los matices migratorios surgen a partir de los países receptores y los espacios donde se alojan, las relaciones socioeconómicas que encuentran, el uso de las tecnologías de información, así como las estructuras de contacto que instauran para sostener comunicación con sus redes homofilicas y heterofilicas en Venezuela. Así como todo el espectro de nación es afectado por el fenómeno de la diáspora, las fronteras tienden a "recalentarse" en sus propias condiciones que las hacen espacios "bi 0 multinacional" con particularidades que irradian a las localidades y ciudades en frontera.

\section{Las regiones y espacios transfronterizos}

Las aproximaciones conceptuales sobre "frontera" intentan considerar las condiciones históricas, políticas y sociales que este término implica. Asumiendo la definición conceptual de Foucher (1991), las fronteras son estructuras espaciales que tienen por función una discontinuidad geopolítica y de marca, de punto de ubicación, sobre registros de lo real, lo simbólico y lo imaginario. Esta postura interpretativa permite la inclusión de un juego propio de relaciones que surgen y que refuerzan dinámicas diferenciadas del resto de un país. Con una distinción adicional y es que a lo largo de esas curvilíneas político-administrativas también existen cambios étnicos, sociales, culturales, económicos y de otro orden que conllevan a que 
las fronteras en sí mismas también cambian. Se trata de espacios sociales que presentan un vínculo geográfico multipolar, en lugar de uno claramente exclusivo, en el caso de los espacios transfronterizos que existen a partir de los límites de Venezuela con sus países vecinos, el fenómeno de la diáspora genera en la actualidad, alteraciones que progresivamente están afectando, los modos y prácticas de vida y en consecuencia, desfasan las líneas de acción gubernamental, intergubernamental e interorganizacional.

Lo anterior, debido que la región transfronteriza está gobernada e intervenida a nivel político y administrativo por distintos sujetos: por los estados colindantes, cuyas políticas determinan el grado de cierre/apertura del límite fronterizo; por eventuales entidades supranacionales, que tienen un papel clave en los procesos de integración del territorio; por los municipios y los gobiernos intermedios situadas en el área de frontera, que administran las relaciones cotidianas que puedan llevarse a cabo alrededor del límite fronterizo; por eventuales transfronterizas, específicamente, encargadas de la gestión del área, que pueden ser constituidas a nivel interestatal, interregional o local.

Además, en una región transfronteriza participan distintos actores del sector privado que interaccionan en ella. La gestión de los límites fronterizos y la cooperación transfronteriza, son ejemplos de gobernanza a múltiples niveles: vertical puesto que existe una división de funciones entre los diferentes niveles institucionales y hay una interacción entre ellos, y horizontal, debido a la participación de los distintos actores de los territorios (Castrillo, 2018).

Las dinámicas fronterizas instauran y, a su vez, exigen propias estrategias de promoción del desarrollo económico y social en lo local. Más allá de la planificación a nivel de país, las consideraciones expuestas, se convierten en exigencias para pensar y planificar estados de bienestar en ciudades y localidades pertenecientes a regiones transfronterizas. En el caso venezolano, la gobernanza es una variable con tinte utópico ante situaciones de diáspora sin administración de los recursos, o de muy largo alcance, si no se incluye lo que sucede en las fronteras a nivel de los venezolanos que emigran y los países vecinos que los reciben formal 0 informalmente.

\section{Maracaibo: retos para una ciudad venezolana afectada por la diáspora}

La evolución de la dinámica socio-económica de Maracaibo pudiese estar relacionada con ciertas condiciones de su fundación y la concepción como una ciudad de tránsito de mercancías y servicios en una economía de puertos, dada su equidistancia entre ciudades mayores durante la época post-colombina. En apenas el 0,78\% de la extensión geográfica del estado Zulia, alcanza el segundo lugar como ciudad de rango poblacional en Venezuela. Derivada de su historia y ubicación estratégica, pudiese ser considerada más allá de su división política administrativa como un entorno territorial articulado con las ciudades menores de San Francisco, Cabimas, Santa Cruz de Mara y La Concepción, y con flujos de intercambio con las ciudades orientales colombianas de Maicao, Riohacha, Valledupar, así como algunas islas cercanas en el Caribe, entre otras, que propician el intercambio comercial de bienes y/o servicios.

De acuerdo con Torres (2011), los resultados dispares de los diversos indicadores socio-económicos de Maracaibo, son derivados en gran parte por: Concentración poblacional superior con movimientos migratorios sin control, que generan desequilibrios territoriales con efectos negativos de tipo ambiental, con degradación de espacios de urbanismo y resguardo de 
Estrategias de sostenibilidad en ciudades fronterizas afectadas por la migración. El caso de la ciudad de Maracaibo

ecosistema lacustre; Crecimiento de la demanda de servicios básicos superior a la inversión en plataforma de servicios, obviamente sin responder a criterios de inteligencia territorial ${ }^{1}$; Limitada capacidad competitiva en términos comparativos con otros entornos locales de Venezuela de acuerdo a factores de condicionamiento para el desarrollo local, y finalmente; Debilidad territorial, expresada en el bajo nivel de relaciones interorganizacionales, el entorno local de Maracaibo y las ciudades medias cercanas que concentran las actividades económicas compartidas.

Desde el enfoque teórico del desarrollo local y a partir del estudio de las condiciones locales del entorno de Maracaibo, se infieren ámbitos problemáticos para generar y capacidades del aprovechamiento sostenible de los recursos disponibles y potenciales, que puedan dirigirse hacia la satisfacción de las necesidades y los problemas básicos de la población. A partir de estas consideraciones, es necesario estudiar si el entorno local de Maracaibo, pudiese ser pensado en términos de formular y ejecutar políticas públicas y privadas que configuren las articulaciones de las potencialidades y limitaciones territoriales.

Desde la perspectiva de redes, se consideró teóricamente la referencia de relaciones interorganizacionales de tipo reticular, como base para un entorno local innovador en el cual sea propicia la interacción de las diversas organizaciones e instituciones participantes de su sistema productivo. Asumiendo además, los postulados teóricos acerca de las redes sociales, que plantean inicialmente la participación social e intercambios en las retículas para la obtención de beneficios individuales con retorno que luego puedan potenciarse a través del número de conexiones (Lin, 2001). En este caso, se evidencian limitadas condiciones que promuevan las relaciones en términos de redes en el entorno local de Maracaibo. De hecho, en términos de afiliación o pertenencia a grupos de contactos estables y con frecuencia de interacción periódica, los flujos migratorios en la actualidad están mutando las formas de relacionamiento y trastocando las prácticas socio-productivas.

De acuerdo con la debilidad determinada en la retícula de relaciones interorganizacionales en el entorno local de Maracaibo, el proceso cognitivo puede estar restringido a lo necesario, en cuanto a las informaciones compartidas para el sostenimiento de las actividades productivas y la obtención de recursos. Las informaciones se comparten en términos parciales y están dirigidas fundamentalmente a conocer el entorno para la identificación de oportunidades. Como canales de comunicación que transmiten informaciones fiables, son utilizados principalmente los contactos homofílicos y heterofilicos, a través de las relaciones interorganizacionales. Los niveles de veracidad de las informaciones suministrados por estos agentes es alto, si se consideran incidentes en los procesos de decisión para emigrar.

No obstante, el ciclo de intercambio es asimétrico y la baja densidad de las relaciones interorganizacionales no logra homogeneizar las informaciones, por lo cual se convierten en realidad en ventajas para aquellos agentes que dispongan de mayor cantidad y calidad de recursos informacionales y los incorporen en sus acciones. Ante contracción de la retícula de relaciones es de esperar menores condiciones para el aumento de los niveles cognitivos de los agentes locales. Asumiendo los planteamientos teóricos de Burt (2004), la baja densidad de vínculos afectivos en lo concerniente a las decisiones de migración implica: poca equivalencia

\footnotetext{
${ }^{1}$ La ciudad sigue creciendo dada sus condiciones geográficas de planicie y la fuerza inercial de su condición histórica en las actividades petroleras de mayor crecimiento en el periodo 1960-1980.
} 
estructural de los estatus relacionados y por ende en sus conductas de inversión, patrones de difusión de informaciones desarrollados a partir de los errores aprendidos colectivamente y bajo número de personas vinculadas directamente.

A partir de la caracterización de los estados, relacionados con los niveles de reticularidad en las relaciones interorganizacionales: confianza, reciprocidad y valoración a las normas; como expresiones de capital social para la planificación del desarrollo económico-social de Maracaibo, es necesario asumir como restricciones de interconectividad las siguientes trayectorias de relacionamiento: Limitadas relaciones sociales en red/bajo nivel de confianza/débil entorno institucional; Limitadas relaciones sociales en red/bajo nivel de difusión de conocimiento/escasa difusión tecnológica/insuficientes innovaciones locales/débil entorno institucional; Limitadas relaciones sociales en red/escasa asociatividad/limitada difusión de información económica/emprendimiento económico no productivo/inversiones rentistas de corto plazo/sistema productivo local tradicional y rentístico.

Tales fracturas en las interconectividades pudiesen considerarse como restricciones para generar capital social en y, a su vez, incidir de forma negativa en el proceso de aprendizaje colectivo con contenido de información para la promoción de estrategias de sostenibilidad para el desarrollo territorial de Maracaibo. Con base en estos resultados se propone el modelo de planificación para el desarrollo territorial de la ciudad fronteriza de Maracaibo, en el marco de la situación de diáspora.

\section{Modelo de planificación para el desarrollo territorial de Maracaibo}

El desarrollo económico local en el entorno espacial de Maracaibo -afectado por los procesos migratorios- estaría en función de contemplar actuaciones en: la estructura productiva; el mercado de trabajo; la capacidad empresarial y tecnológica; los recursos socio-productivos; el sistema de financiamiento; la estructura social y política; el patrimonio histórico y cultural; principalmente afectados por el fenómeno de diáspora. Dichos factores exigen estrategias coherentes y sustentables. En especial, una condición determinante es la actuación del gobierno local en el proceso de construcción de inteligencia territorial, concebido como agente de cohesión entre los diversos agentes locales, con visión prospectiva y competencias organizativas, capaz de aprovechar sus competencias administrativas y jurídicas para impulsar y sostener actuaciones conjuntas del resto de agentes locales y los requeridos externamente por el entorno.

La proposición de un modelo de planificación que incida en la generación y dinamización de cambios socio-productivos en Maracaibo, exige la previa identificación y aceptación de lineamientos ${ }^{2}$ acerca de la concepción de desarrollo económico y social que se aspira alcanzar para el entorno local, en el cual pueda materializarse, siguiendo a Vázquez Barquero (1988), un crecimiento que afecte a todos los agentes sociales territorialmente definidos, y que se concrete en mejoras de los niveles de vida de sus habitantes. Los lineamientos de orientación para la endogeneidad del desarrollo que se asumen son:

\footnotetext{
2 Iniciativa Global ART - Articulación de Redes Territoriales y Temáticas de Cooperación para el Desarrollo Humano, concebida como interfaz del Bureau de Partenariados (BP) del Programa de las Naciones Unidas para el Desarrollo (PNUD) para aquellos socios de la cooperación internacional interesados en armonizar sus respectivas acciones en apoyo a las estrategias nacionales de desarrollo local y convencidos de que el enfoque territorial valoriza el rol de las comunidades territoriales, facilita la complementariedad entre los diferentes actores del desarrollo en los países y materializa el potencial estratégico de la cooperación descentralizada, mejorando así la efectividad y eficacia del apoyo a los procesos de desarrollo priorizados por los paises mismos.
} 
Estrategias de sostenibilidad en ciudades fronterizas afectadas por la migración. El caso de la ciudad de Maracaibo

1) El desarrollo de un territorio está fuertemente condicionado por la voluntad de asumir compromisos de largo plazo por sus agentes sociales; 2) La cohesión y los acuerdos mediante consensos hacia los objetivos de bienestar, promueve la coordinación de las acciones de apoyo, las políticas y las estrategias consideradas en los esfuerzos de planificación y gestión del desarrollo local; 3) El desarrollo de un territorio gira alrededor de la valorización de las potencialidades endógenas; 4) El desarrollo del territorio exige promover redes socioproductivas; 5) El desarrollo depende de la capacidad de integrar las iniciativas empresariales en un ambiente que facilite su concreción y su crecimiento; 6) El territorio debe disponer de instrumentos adecuados para la aplicación de políticas y de estrategias de desarrollo local; y 7) El territorio es un sistema totalmente abierto.

La construcción de la visión prospectiva para el entorno de Maracaibo, conduce a la identificación de la Gobernanza local como la variable de mayores correspondencias con los lineamientos de orientación para el desarrollo económico y social en lo local y, a su vez, con fuertes incidencias para la transformación de la realidad actual. Recurriendo a las características sociales del entorno territorial de Maracaibo -incluyendo las fronterizas-, los procesos y acciones que puedan instaurar bases para la sostenibilidad, exigen como condiciones:

1) Disposición activa de inicio por parte de un gobierno local que involucre los agentes sociales de acuerdo principalmente a su naturaleza y pertinencia con los objetivos declarados, planes y políticas en pro del desarrollo económico y social; 2) Coherencia en las declaraciones y acciones por parte del gobierno local para ser asumidas y utilizadas como mensajes de confianza por el resto de agentes sociales involucrados en el entorno local; 3) Privilegio a las relaciones interorganizacionales e interconectividades en disposición de retículas como estrategias de acción local; 4) Homogeneidad de información por ámbito, nivel y sector en lo referente a las actuaciones institucionales; 5) Administración transparente de los recursos involucrados en los planes programas y proyectos de acción local.; 6) Equidad en la distribución de responsabilidades, esfuerzos y beneficios por naturaleza de agente social involucrado; y 7) Búsqueda permanente de consensos a través de la comunicación y el diálogo.

Dichas condiciones aceleran la generación de confianza institucional e interorganizacional para la gobernanza, fundamentalmente para las fases de planificación y gestión del desarrollo económico y social, superando el enfoque participativo tradicional y facilitando las relaciones interorganizacionales hacia alianzas locales de mayor plazo y alcances de aprendizajes colectivos. De lograrse, se justifica su rango de variable clave para el futuro de un desarrollo económico y social que ocurra en el entorno de Maracaibo, y a partir de ella se instaura la dimensión nuclear de un modelo de promoción del desarrollo sostenible.

La propuesta de planificación del desarrollo territorial y sostenible para una ciudad con naturaleza fronteriza consiste en una estructura de anillos concéntricos, desarrollado por Torres (2011), que tiene como propósito, alcanzar la configuración de un sistema que comparta un mismo núcleo de origen, en este caso constituido por la declaración de gobernanza territorial local a partir de la cual se expandan retículas mediante capas de agentes sociales interrelacionados. La función clave del núcleo de gobernanza es garantizar la comunicación homogénea con la totalidad de las capas reticulares y administrar el sistema, aportando de esta forma, factibilidad y viabilidad en los planes, programas y proyectos pensados y que se gestionen para el entorno. 
Lo anterior es posible si cada círculo concéntrico, y a su vez reticular, se construye y dispone sobre la complexión del anterior. De tal manera, que entre ellos debe existir una elevada porosidad con la finalidad de facilitar las relaciones dinámicas y sobre todo abiertas entre los agentes sociales integrantes para el intercambio de información con contenidos de valor agregado. La superposición de capas de relaciones entrama un tejido socio-productivo más conectivo en términos de relaciones interorganizacionales. La estructura de relaciones reticulares y concéntricas favorece la expansión de las relaciones horizontales sin intervenir en aspectos característicos y propios de los ámbitos, niveles y sectores existentes en el entorno territorial y el espacio colindante en lo fronterizo de Maracaibo.

Básicamente, el ordenamiento de los agentes sociales sería en razón de promover coherencia en decisiones de incidencia territorial en lo local e incrementar los niveles de confianza para relaciones vinculativas, asociativas y cooperativas que puedan establecerse. A continuación se describen los dos primeros anillos de retículas concéntricas considerados en el modelo teórico-operativo para la promoción de fuerzas dinamizadoras del desarrollo económico y social en Maracaibo. De articularse, estarían generando atractores para nuevas redes socioproductivas.

\section{Primer anillo de relaciones interorganizacionales}

La articulación de los intereses y posiciones en correspondencia con las variables motrices del ejercicio prospectivo y de acuerdo a la valoración de lo territorial, exigen que se entramen y configuren redes de relaciones interorganizacionales para la planificación y gestión del desarrollo territorial del entorno a partir de un primer anillo concéntrico, considerado estratégico e integrado por la siguiente retícula primaria en torno al gobierno local: Comunidades, empresas locales, entidades financieras y universidades.

En el caso de las comunidades, son agentes sociales considerados como beneficiarios directos de las acciones de cambio local y quienes manifiestan en sí mismos los resultados del desarrollo económico y social. Constituyen un actor político fundamental del desarrollo y la gobernabilidad, que puede asumir compromisos y responsabilidades, dialogando activamente con las estructuras centrales del Estado y las organizaciones internacionales. Las empresas locales, por su parte, son unidades socio-productivas ubicadas en la ciudad y con ámbitos geográficos de mercado básicamente local, las cuales constituyen la plataforma empresarial distribuida en el entorno.

Mientras que las entidades financieras ubicadas en el entorno local, constituyen entes cuya naturaleza es clave en el financiamiento de actividades públicas y/o privadas, de carácter natural y/o jurídico en el entorno local. Finalmente, las universidades con actuaciones en el entorno local, son instituciones de educación superior, fundamentales en la transversalidad del conocimiento y la promoción de actitud proclive al aprendizaje y la difusión de innovaciones.

La configuración de esta primera retícula pudiese considerarse de carácter atomizante, pues sería determinante para la instauración de mecanismos propiciadores de confianza institucional, que inicialmente formalizados a partir de la Declaración de Maracaibo, con actuaciones recíprocas incidan posteriormente en generar en tendencia sostenida niveles de confianza interorganizacional.

La selección de los agentes integradores de la retícula base, parte en primera instancia de los resultados del análisis prospectivo de actores, en el cual son ubicados como los de mayor 
Estrategias de sostenibilidad en ciudades fronterizas afectadas por la migración. El caso de la ciudad de Maracaibo

incidencia y movilidad hacia el logro de los objetivos estratégicos y, por ende, del escenario apuesta y, en segundo lugar, por sus implicaciones directas en las fuerzas dinamizadoras del desarrollo económico y social en lo local. En tal sentido, se requiere: Por una parte, cambio y adaptación institucional, además de organización social para el desarrollo; conducido desde el gobierno local en lo concerniente a la proposición de instituciones que privilegien el entorno de Maracaibo y el principio de coherencia de las estrategias y políticas planificadas y gestionadas desde los distintos niveles de responsabilidad para la atención integral de los habitantes. Por otra parte, una organización flexible de la producción; desarrollo urbano del territorio y difusión de las innovaciones y el conocimiento, con el involucramiento directo de la retícula primaria.

De igual forma, el entramado nuclear pudiese acelerar medios para la transmisión y amplificación al resto de agentes sociales en el entorno local, ineludible para lograr niveles básicos de cohesión que la haga sostenible en los diferentes ámbitos, niveles y sectores ${ }^{3}$. Tal propósito hace necesario la creación como componente institucional del gobierno local, de una agencia de desarrollo local, intencionalmente responsable para efectuar y actualizar el pensamiento prospectivo acerca de la gestión del desarrollo local y en consecuencia de las actuaciones de alcance territorial para el entorno de Maracaibo.

\section{Segundo anillo de relaciones interorganizacionales}

El segundo aro de la estructura concéntrica propuesta para la promoción de fuerzas dinamizadoras del desarrollo económico social en Maracaibo, incorpora una categoría de agentes sociales ubicados como potencialmente determinantes de acuerdo a los resultados del análisis prospectivo: Consejo legislativo local; cuerpos de gestión comunal; Gobierno regional; Dirección de Desarrollo Económico Regional y medios de comunicación. Considerados como claves para viabilizar los acuerdos formales generados de base para la Gobernanza territorial local, las actuaciones de la agencia de desarrollo local y para expandir a través de la reciprocidad en las relaciones interorganizacionales, las retículas socio-productivas en el entorno de Maracaibo.

Las incidencias de esta segunda capa de retícula serían fundamentales en el alcance de cadenas productivas locales; fuentes de financiamiento a nuevos empresarios; sistema de información de mercados; estrategias de atracción de inversión local; capital humano local y sistema de educación local. De articularse el segundo anillo de redes, se generaría mayor participación e involucramiento de los agentes sociales para componentes estructurales básicos en los planes, programas y proyectos de gestión local para el desarrollo; específicamente: construcción y modernización de infraestructuras urbanas y de servicios, sistema de financiamiento a capitales de riesgo, sistema de información de mercados, sistema de ordenamiento urbano territorial, formación y participación ciudadana y comunitaria, uso de tecnologías de información y comunicación, gestión ambiental y promoción tecnológica.

En esta segunda capa de relaciones interorganizacionales, la creación de un centro de relaciones internacionales para Maracaibo y un sistema de incubación de empresas locales,

\footnotetext{
${ }^{3}$ De no efectuarse respuestas positivas entre los agentes de la retícula base, la participación se reducen a formalismos que caducan con la ausencia de acciones de cambio local.
} 
potenciarian las relaciones interorganizacionales y las capacidades organizativas locales para promover las actividades productivas.

\section{Estrategias de sostenibilidad para el desarrollo territorial}

La construcción del escenario apuesta para Maracaibo como ciudad frontera y en el contexto de diáspora, exige consideraciones estratégicas y prospectivas, que viabilicen la planificación y gestión local e impulsen las fuerzas dinámicas hacia un desarrollo económico y social localizado. Dichas consideraciones requieren ser instauradas a partir de las acciones del gobierno local, y posteriormente fortalecidos los agentes sociales incidentes de forma directa y potencial en el modelo teórico-operativo. Las consideraciones estratégicas de primer orden deben establecer: Los principios orientadores, que constituyan los valores éticos, ineludibles en el ejercicio público para promover transparencia y los propósitos de igualdad en el beneficio social.

Los desafíos prospectivos deben ser compatibles con las fuerzas dinamizadoras del desarrollo endógeno, territorial y sustentable en lo local y hacer posible la construcción de la red lógica de pertinencias con los programas, proyectos y acciones centralizadas. Se asumen para Maracaibo: cambio y adaptación institucional, innovación y difusión del conocimiento, organización flexible para el emprendimiento, desarrollo urbano del territorio y organización Social para el Desarrollo. Algunas bases referenciales para la declaración de desafíos prospectivos, estrategias, programas y proyectos, que puedan ser consideradas por el gobierno local y las redes socio-productivas contempladas en el modelo teórico-operativo, para dinamizar las fuerzas del desarrollo económico y social en Maracaibo, son las que a continuación se presentan (Ver cuadros 1 y 2 ).

Cuadro No. 1.

Desafíos prospectivos I, II y III

\begin{tabular}{|l|}
\hline \multicolumn{1}{|c|}{ I. Desafío prospectivo: MARACAIBO EMPRENDEDORA EN RED } \\
\hline Estrategias base \\
\hline Formular políticas municipales para el apoyo de Pymes locales. \\
\hline $\begin{array}{l}\text { Fortalecer y diversificar las modalidades de relación entre el sector empresarial y las } \\
\text { comunidades. }\end{array}$ \\
\hline Formular e implantar medios de atracción de inversión sostenida para Maracaibo. \\
\hline Diseñar medios de estímulo para las relaciones empresariales y las exportaciones. \\
\hline Fomentar la actividad turística en Maracaibo. \\
\hline Diversificar bases de apoyo local para nuevos sectores económicos. \\
\hline Diseñar líneas de financiamiento para nuevos emprendedores. \\
\hline \multicolumn{1}{|c|}{ Desafío prospectivo: MARACAIBO, ClUDAD INNOVADORA } \\
\hline Estrategias base \\
\hline $\begin{array}{l}\text { Fortalecer el impulso de los procesos de generación, gestión y transferencia del } \\
\text { conocimiento aprovechable. }\end{array}$ \\
\hline $\begin{array}{l}\text { Fortalecer la creación de comunidades de aprendizaje en la investigación para consolidar el } \\
\text { desarrollo de una cultura científica y tecnológica. }\end{array}$ \\
\hline $\begin{array}{l}\text { Impulsar el desarrollo de áreas científico-tecnológicas para la integración de proyectos con } \\
\text { aliados estratégicos. }\end{array}$ \\
\hline
\end{tabular}


Estrategias de sostenibilidad en ciudades fronterizas afectadas por la migración. El caso de la ciudad de Maracaibo

\begin{tabular}{|l|}
\hline $\begin{array}{l}\text { Fomentar desde las universidades el incremento de la capacidad en la prestación de } \\
\text { servicios tecnológicos, para la intervención del territorio a través de proyectos locales. }\end{array}$ \\
\hline $\begin{array}{l}\text { Fomentar el desarrollo de investigaciones sobre los impactos de las actividades asociadas } \\
\text { al turismo sostenible. }\end{array}$ \\
\hline III. Desafío prospectivo: MARACAIBO, GENTE PARA EL DESARROLLO \\
\hline Estrategias base \\
\hline Promover consenso entre los agentes sociales locales para la gestión de proyectos. \\
\hline Fortalecer las relaciones interorganizacionales para la gestión de iniciativas. \\
\hline Fortalecer la cultura local hacia la seguridad jurídica, confianza en las. \\
\hline $\begin{array}{l}\text { Promover acciones centradas en el aprendizaje social como agente dinamizador de las } \\
\text { organizaciones ante los desafíos del desarrollo local. }\end{array}$ \\
\hline
\end{tabular}

Fuente: Elaboración propia.

Cuadro No. 2.

Desafíos prospectivos IV, $\mathrm{V}$ y $\mathrm{VI}$

\begin{tabular}{|l|}
\hline \multicolumn{1}{|c|}{ I. Desafío prospectivo: MARACAIBO, ENTORNO DE CONSENSO Y PAZ } \\
\hline Estrategias base \\
\hline $\begin{array}{l}\text { Diseñar un modelo de organización y gestión pública descentralizada, con capacidad de } \\
\text { adaptación a los retos del desarrollo económico local. }\end{array}$ \\
\hline $\begin{array}{l}\text { Fortalecer la capacidad de gestión de las instituciones públicas locales para promover } \\
\text { iniciativas económicas locales, sustentables y competitivas. }\end{array}$ \\
\hline Diseñar modelos gerenciales flexibles de gestión pública. \\
\hline Fortalecer las modalidades de relación entre los agentes de los anillos de desarrollo local. \\
\hline $\begin{array}{l}\text { Fortalecer un marco institucional sólido y confiable con capacidad para orientar y dinamizar } \\
\text { con eficiencia y eficacia un proceso de desarrollo local. }\end{array}$ \\
\hline $\begin{array}{l}\text { Diseñar y promover la creación de estructuras organizativas basadas en el fortalecimiento } \\
\text { de capacidades, asociaciones, vínculos, cooperación y empoderamiento en Maracaibo. }\end{array}$ \\
\hline Establecer mecanismos de control de la gestión local. \\
\hline $\begin{array}{l}\text { Establecer mecanismos de seguimiento y evaluación de los programas y proyectos de } \\
\text { desarrollo local. }\end{array}$ \\
\hline \multicolumn{1}{c|}{ Desafío prospectivo: MARACAIBO, HABITAT PARA LA VIDA } \\
\hline Estrategias base \\
\hline $\begin{array}{l}\text { Promover la innovación y el emprendimiento para el fortalecimiento de las capacidades } \\
\text { locales en materia de manejo del hábitat, seguridad alimentaria, diversificación de fuentes } \\
\text { de energía, seguridad y defensa, manejo de biodiversidad y mejoramiento de los espacios } \\
\text { urbanos. }\end{array}$ \\
\hline $\begin{array}{l}\text { Incentivar la concienciación de los agentes sociales de Maracaibo para el hábitat y el turismo } \\
\text { sostenible. }\end{array}$ \\
\hline
\end{tabular}

Fuente: Elaboración propia

La endogeneidad local de Maracaibo como una ciudad frontera y con límites territoriales de alcance plurinacional, exige procesos sociales, económicos, científicotecnológicos, políticos, administrativos, entre otros necesarios para instaurar un marco institucional propicio para la interacción de los agentes en términos de redes socio-productivas que sostengan relaciones con contenidos de innovación y emprendimiento, al reducirse los costos de transacción por relacionarse. El citado fortalecimiento institucional es condición sine 
qua non para la gestión de un plan de desarrollo local. La persistencia de instituciones débiles, pueden generar mayores problemas locales: mano de obra calificada sin ubicación laboral; incentivos para la inversión local pero baja productividad y competitividad empresarial; promoción para exportar sin informaciones acerca de mercados externos, por mencionar algunos desaciertos de la planificación y gestión local, enfocada siguiendo estrategias verticales desvinculadas de las bases.

\section{Conclusiones}

La investigación efectuada intentó superar la generación de respuestas, en estricto sentido, económicas y empresariales en la búsqueda de la ocurrencia del desarrollo local; para describir y explicar las condiciones institucionales catalizadoras de lo social y lo económico en los entornos regionales y/o locales, que afecten la conformación de relaciones socio-productivas en redes; claves para intercambios de información, conocimientos y recursos para sostener capacidades innovadoras y procesos de emprendimiento.

El desarrollo económico local contrasta definitivamente con el desarrollo económico a nivel de país por la inclusión de la referencia territorial en el análisis sobre la conformación y niveles de desarrollo alcanzados en las diversas áreas interiores de los países, acentuando los elementos del territorio y sus efectos en las dimensiones económicas, tecnológicas, políticas y sociales.

Es necesario reubicar el concepto de desarrollo en un marco constructivista, subjetivo e intersubjetivo, y, por cierto, endógeno, o sea, directamente dependiente de la autoconfianza colectiva en la capacidad para 'inventar' recursos, movilizar los ya existentes y actuar en forma cooperativa y solidaria, desde el propio territorio. Los enfoques teóricos considerados para la potenciación de sistemas productivos locales son favorables para las consideraciones fronterizas y sensibles a los movimientos migratorios.

Un modelo de planificación del desarrollo territorial y sostenible para ciudades frontera que puedan gestionar los movimientos migratorios, incluso en condiciones de diáspora, puede ser sobre la base de una estructura de anillos concéntricos. El propósito de este tipo de ordenamiento es alcanzar la configuración de un sistema que comparta un mismo núcleo de origen, en este caso constituido por la declaración para la gobernanza territorial local a partir de la cual se expandan retículas mediante capas de agentes sociales interrelacionados. La función clave del núcleo de gobernanza es garantizar la comunicación homogénea con la totalidad de las capas reticulares y administrar el sistema, aportando de esta forma, factibilidad y viabilidad en los planes, programas y proyectos pensados y que se gestionen para el entorno.

En la medida que se logren articular ambos anillos concéntricos en retículas de relaciones que aprovechen y fortalezcan las capacidades internas de los agentes sociales del entorno local, de instaurar bases de confianza y respuestas positivas en las interacciones socioproductivas, las probabilidades de mejorar las instituciones necesarias para un clima propicio para la expansión aglutinante y progresiva del resto de agentes sociales, disponiéndose en mayores capas concéntricas y dinámicas relaciones interorganizacionales.

La evolución de la dinámica socio-económica de Maracaibo pudiese estar relacionada con ciertas condiciones de su fundación y la concepción como una ciudad de tránsito de mercancías y servicios en una economía de puertos, dada su equidistancia entre ciudades mayores durante la época post-colombina. 
Partiendo de la premisa de un futuro por construir y explorando hipótesis factibles que puedan incidir en el desarrollo de un territorio, es posible lograr la interacción de los agentes sociales y en especial de los responsables de las políticas de gobierno para establecer una nueva gobernanza del territorio local en condiciones fronterizas.

\section{Referencias Bibliográficas}

Arango, Joaquín. (1985). Las Leyes de las Migraciones de E. G. Ravenstein, cien años después. En Revista Española de Investigaciones Sociales (REIS), No. 32. España. (Pp. 7-26).

Aruj, Roberto (2008). Causas, consecuencias, efectos e impacto de las migraciones en Latinoamérica. Papeles de Población, Vol. 14, № 55, enero-marzo, México (Pp. $95-$ 116).

Baptista, Asdrúbal. (2005). El capitalismo rentístico. Elementos cuantitativos de la economía venezolana. En Cuadernos del Cendes. Volumen 22, número 60. Venezuela. (Pp. 95-111).

Blanco, Cristina. (2000). Las migraciones contemporáneas. Ciencias Sociales, Alianza Editorial. España.

Burt, Ronald. (2004). Social Contagion and Innovation: Cohesion versus structural equivalence. Social networks: critical concepts in sociology. Volumen 4. Routledge. USA. (Pp. 1287-1335).

Castrillo, Leonor. (2018). Escenarios de integración de las universidades de frontera en el proceso de internacionalización. Tesis Doctoral. Doctorado en Ciencias Sociales, mención Gerencia. Facultad de Ciencias Económicas y Sociales. Universidad del Zulia. Venezuela.

Fernández, Mercedes (2013). Aproximación interdisciplinar al análisis de las migraciones. Universidad Pontificia Comillas. España.

Foucher, Michel (1991), Fronts et frontières, un tour du monde géopolitique, 2 ed, Fayard, Francia.

Lin, Nan. (2001). Social Capital, A Theory of Social Structure and Action.Cambridge University Press. Cambridge. USA.

Massey, Douglas S. et al. (1998). Worlds in motion. Understanding International Migration at the End of the Millennium. Clarendon Press. Inglaterra.

Torres Granadillo, Fernando J. (2011). Capital social, emprendimiento e innovación en la sostenibilidad del desarrollo económico social en lo local. Tesis Doctoral. Doctorado en Ciencias Humanas. Universidad del Zulia. Venezuela

Vázquez Barquero, Antonio. (2005). Las nuevas fuerzas del desarrollo. Antoni Bosch Editor, S.A. España. 